\title{
Drop Tower Experiment to Study the Capillary Flow in Symmetrical and Asymmetrical Channels: Experimental Set-up and Preliminary Results
}

\author{
Xiao Liang Chen ${ }^{1}$ Yuan Gao ${ }^{1}$ Q Qiu Sheng Liu ${ }^{1,2}$
}

Received: 17 March 2016 / Accepted: 13 July 2016 / Published online: 1 August 2016

(c) Springer Science+Business Media Dordrecht 2016

\begin{abstract}
Owing to the development of the space exploration activities, the in-orbit management of fluids such as the transportation of propellant liquid in microgravity becomes the important direction of microgravity fluid research, and one of main problems is the stability behaviors of free surface flow in capillary channel of PMD. In the present study, an experiment set-up of the fluid transport with two different capillary channels has been developed on the Beijing Drop Tower platform. Both symmetrical and asymmetrical flow channels, with the same cross-sectional areas and lengths and different cross-sectional geometries were used and HFE-7500 is chosen as test liquid. 10 times of the drop-down experiments were performed for investigation of the capillary flow characters in different volumetric flow rates, and the three main patterns of capillary flows: subcritical flow, critical flow and supercritical flow were found in experiments, these patterns are distinguished by the movement of the point of lowest surface over time. Meanwhile, the critical flow rates at which free surface becomes instable observed in our experiments are
\end{abstract}

This article belongs to the Topical Collection: Advances in Gravityrelated Phenomena in Biological, Chemical and Physical Systems Guest Editors: Valentina Shevtsova, Ruth Hemmersbach

Qiu Sheng Liu

liu@imech.ac.cn

1 Key Laboratory of Microgravity, Institute of Mechanics, Chinese Academy of Sciences, Beijing, 100190, China

2 School of Engineering Science, University of Chinese Academy of Sciences, Beijing, 100049, China
(1) $2.7 \pm 0.2 \mathrm{ml} / \mathrm{s}$ for the critical flow rate of asymmetrical channel; and (2) $2.2 \pm 0.2 \mathrm{ml} / \mathrm{s}$ for symmetrical channel flow, respectively.

Keywords Capillary channel flow $\cdot$ Interface stability · Critical flow rate · Drop tower

\section{Introduction}

The fluid transport process is very important to almost all industries. Especially for liquid management in space and lab-on-chip (Rollins et al. 1985; Haeberle and Zengerle 2007), which provides a condition that the gravity is negligible, unlike the most terrestrial issues, the capillary effort dominates the behavior of the free surface. And the most challenging problem is how to preserve a continuous, bubble-free flow. Once the flow rate within the channel has exceeded a certain value, the bent free surface is of no capacity to maintain a stable state, and the surface collapses and gas ingestion occurs.

Initial work in this field is done by (Jaekle 1991), he compared the collapse of free surface flow of capillary channel flow to the choking of compressible flow within ducts, and established the mathematical model to predict the flow rate limitation. (Rosendahl et al. 2002; Conrath et al. 2013; Haake et al. 2006) and (Klatte 2011; Canfild et al. 2013) conducted the drop tower experiments, sounding rocket flights and international space station experiments, they investigated the influences of channel's length and cross section on the flow behavior, and the critical flow rate is also determined. (Rosendahl et al. 2004; Rosendahl et al. 
Fig. 1 The cross-sectional geometries of two capillary flow channels (a)Cross section of asymmetrical channel; (b) Cross section of symmetrical channel

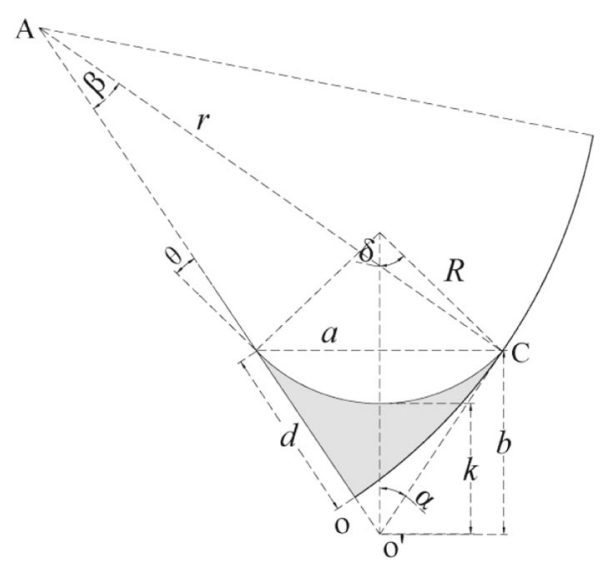

(a)

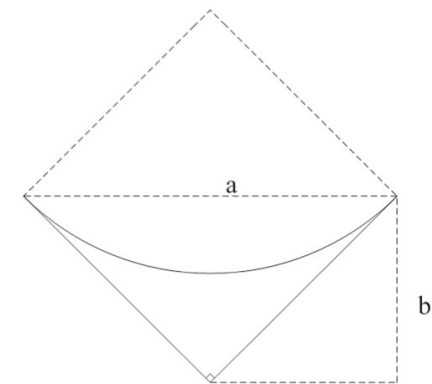

(b)
2010; Wei et al. 2013; Haake et al. 2010; Grah and Dreyer 2010) and (Bronowicki et al. 2015) use one dimensional model and three dimensional simulation method to get critical flow rate value and transition behaviour theoretically. But most of these works foucused on symmetrical capillary channel geometries.

In the real PMD designed for using in space, the liquid transport channels are asymmetrical ones in general, so we choose a typical (a curved vane is perpendicular to a straight vane) asymmetrical channel in present experiment study. The asymmetrical channel test section and symmetrical one (two straight vanes are perpendicular to each other) are intergrated in parallel on the set-up, so we can figure out the capillary flow patterns and analyze the differences of flow stability characters in two different channels

\section{Experimental Investigation}

\section{Apparatus and Procedure}

For the observation of the free surface flow instability behavior and critical flow rate in different channels, a microgravity fluid transport experiment set-up with two capillary flow channels has been developed. The cross-sectional geometries of the two capillary channels are different as shown in Fig. 1. One is a symmetrical channel and orther one is an asymmetrical channel, with same cross-sectional area and same length $(30 \mathrm{~mm})$ of opened-free surface window in flow direction.

The experimental apparatus is shown in Fig. 2, which consists two fluid containers filled with test liquid in two containers, and a gear pump maintains a flow with constant
Fig. 2 Schematic of experimental apparatus

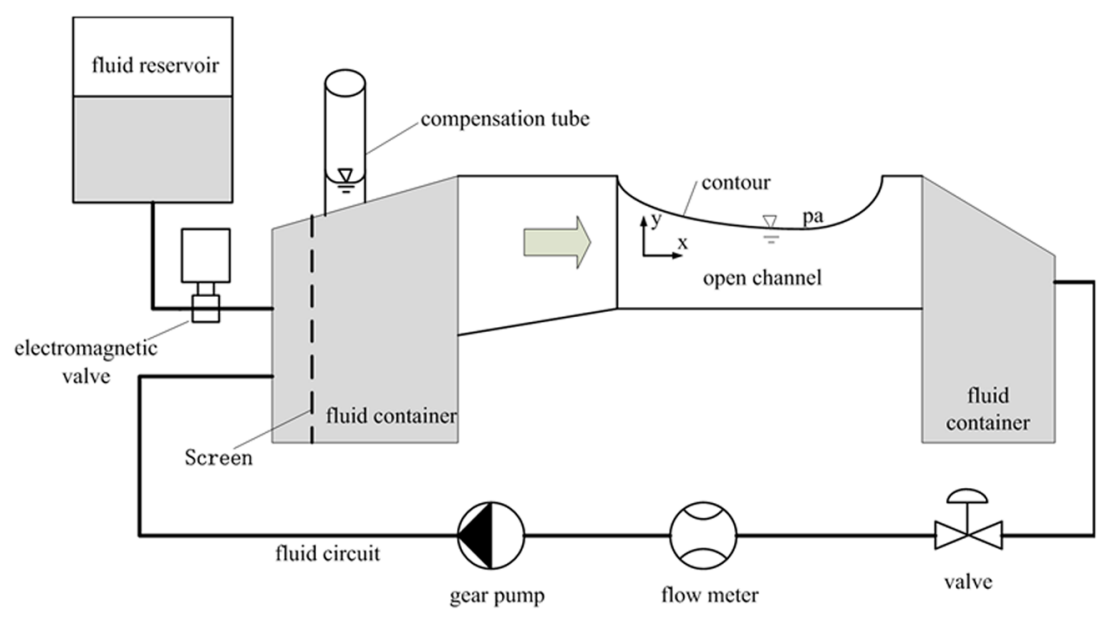


Table 1 The physical properties of HFE-7500 at different temperatures $T$

\begin{tabular}{llll}
\hline$T\left({ }^{\circ} \mathrm{C}\right)$ & $\rho\left(\mathrm{kg} / \mathrm{m}^{-3}\right)$ & $v\left(\times 10^{-6} \mathrm{~m}^{2} \mathrm{~s}^{-1}\right)$ & $\sigma\left(\times 10^{-3} \mathrm{kgs}^{-2}\right)$ \\
\hline 25.0 & $1,620 \pm 2$ & $0.773 \pm 0.1$ & $16.66 \pm 0.1$ \\
30.0 & $1,610 \pm 2$ & $0.715 \pm 0.1$ & $16.16 \pm 0.1$ \\
35.0 & $1,600 \pm 2$ & $0.664 \pm 0.1$ & $15.67 \pm 0.1$ \\
\hline
\end{tabular}

volumetric flow rate from the outlet container to the inlet one, using two valves to make two channels with different volumetric flow rates, which are measured by two flow meters. Two CCD cameras are used with two mirrors for flow observation. We choose HFE-7500 as test liquid, the physical properties of HFE-7500 is given in Table 1, the experiment temperature is about $30^{\circ} \mathrm{C}$ and capsule inside system pressure is $1 \mathrm{~atm}$, the accuracy of flow meter is $0.2 \mathrm{ml} / \mathrm{s}$. The experiment setup within drop capsule is shown in Fig. 3.

\section{Photographic Systems}

Schematic drawing and photo image of the capillary flow channels with optical observation system are shown in Fig. 4. A parallel LEDs backlight source is positioned between two flow channels on the baseboard of the set-up (see in Fig. 4(b) ). Two high-speed and high-resolution cameras are used to capture images of the free surface behavior of the two capillary channel flows. The free surface shapes of the test liquid flow are captured from the side view of capillary flow channels, by a CCD camera at $200 \mathrm{fps}$ with $1280 * 1024$-pixel resolution. Meanwhile, there are two supervision cameras to monitor whether the experiment is

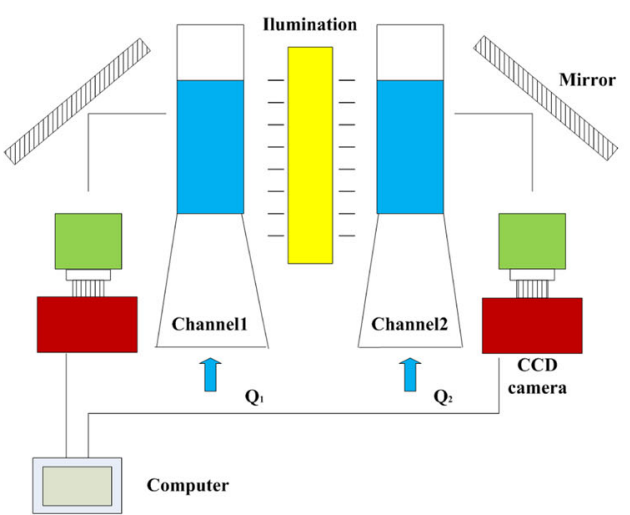

(a)

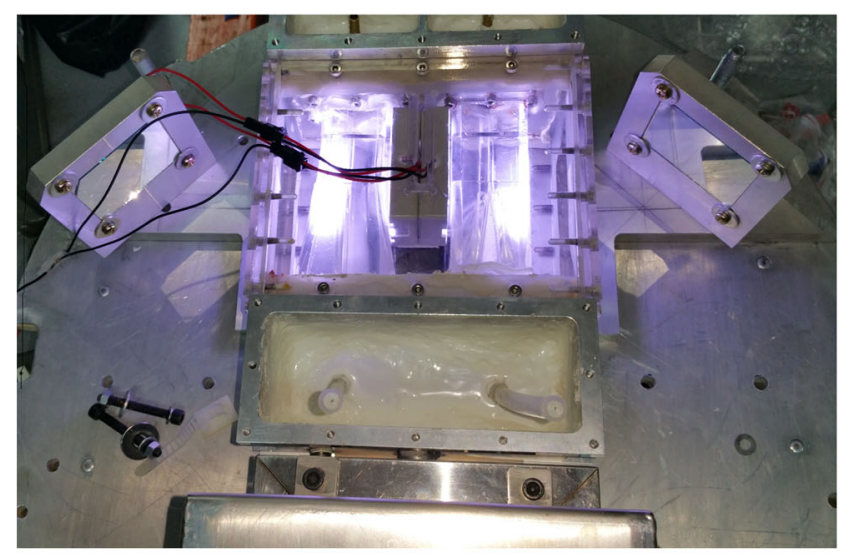

(b)

Fig. 4 (a) Schematic drawing of optical system; (b) Illumined two parallel capillary flow Channels

conducted in right way. The video images of liquid flow in channels are stored in the two cameras during the drop
Fig. 3 Photographs of the experiment set-up (a) Apparatus integrated on the drop capsule; (b) Capillary flow channels and optical system

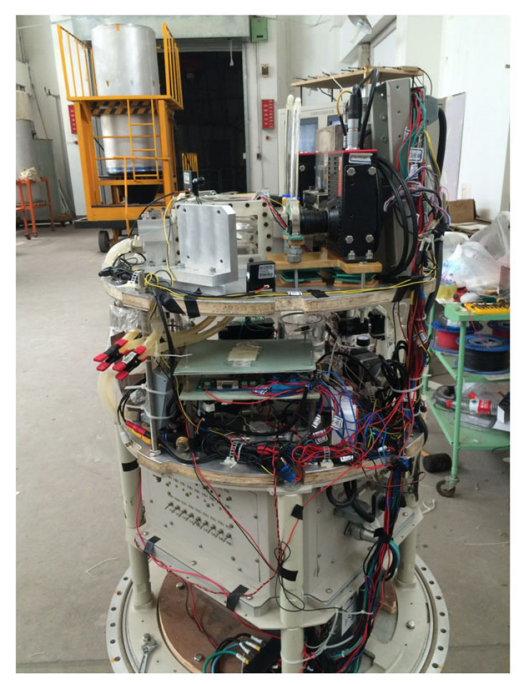

(a)

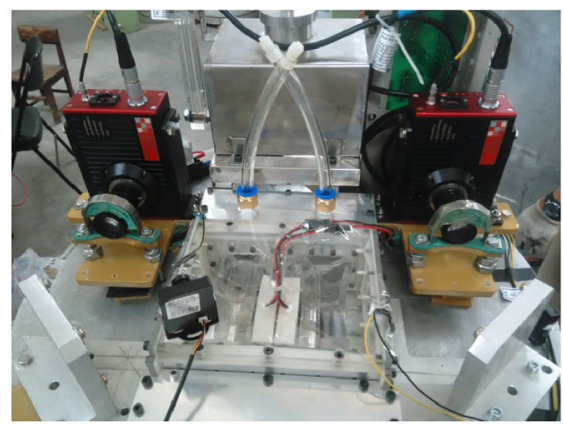

(b) 
Fig. 5 Method to pick up the location of the lowest point of the free surface

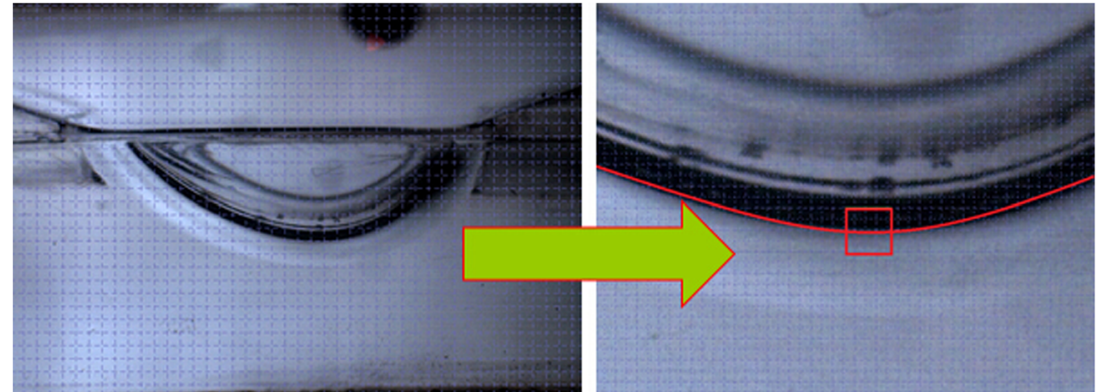

experiment, and the image dates of will be download to our Lap-top computer when the capsule is open after each drop.

\section{Experimental Results Analysis}

The instability behavior of the free surface of capillary channel flow is that the free surface collapses and gas is ingested . In our drop tower experiments, the microgravity time of $3.6 \mathrm{~s}$ is given by dropped capsule. During the last $0.8 \mathrm{~s}$ of the microgravity time when the free surface reaches a steady state for observation, we can pick up the location of the lowest point of the steady free surface and get the coordinates of the lowest points to plot them versus time. The resolution of the measurement is about $0.2 \mathrm{~mm}$, and the method of image analysis is shown in Fig. 5.

The capillary flow in both asymmetrical channel and symmetrical one in microgravity condition will induce the

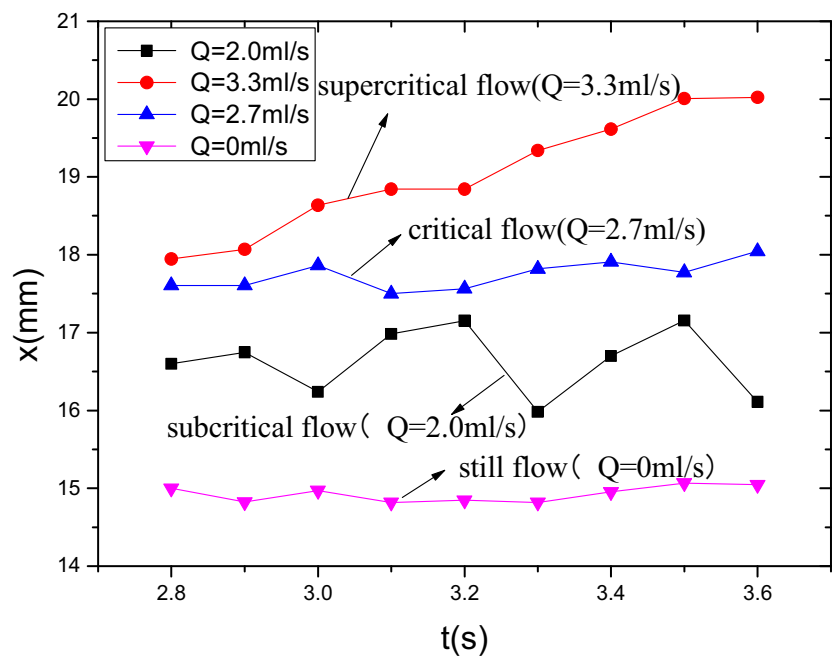

Fig. 6 Variation of the location of lowest point (x direction) of free surface during the microgravity time, for asymmetrical channel deformation of free surface in the open section of the test channel. With the increase of the flow rate, the shape of free surface proceeds to bend further into the channel, and the lowest point of free surface moves forward evidently in flow direction with time at or over a critical value of flow rate. Fig. 6 shows the variation of the coordinates of the lowest point of the free surface in flow direction ( $\mathrm{x}$ direction), in microgravity time for different flow rates in asymmetrical channel. Compared with zero flow rate conditions $\mathrm{Q}=0 \mathrm{ml} / \mathrm{s}$, it was found that there exist three typical flow patterns: subcritical flow, critical flow $\left(\mathrm{Q}_{\text {crit }}=2.7 \mathrm{ml} / \mathrm{s}\right)$ and supercritical flow. These similar behaviors of the capillary flow was also found in the symmetrical channel but the critical flow rate is $\mathrm{Q}_{\text {crit }}=2.2 \mathrm{ml} / \mathrm{s}$, as shown in Fig. 7.

For both test channels in drop microgravity experiment, when the flow rate is smaller than the critical value, the

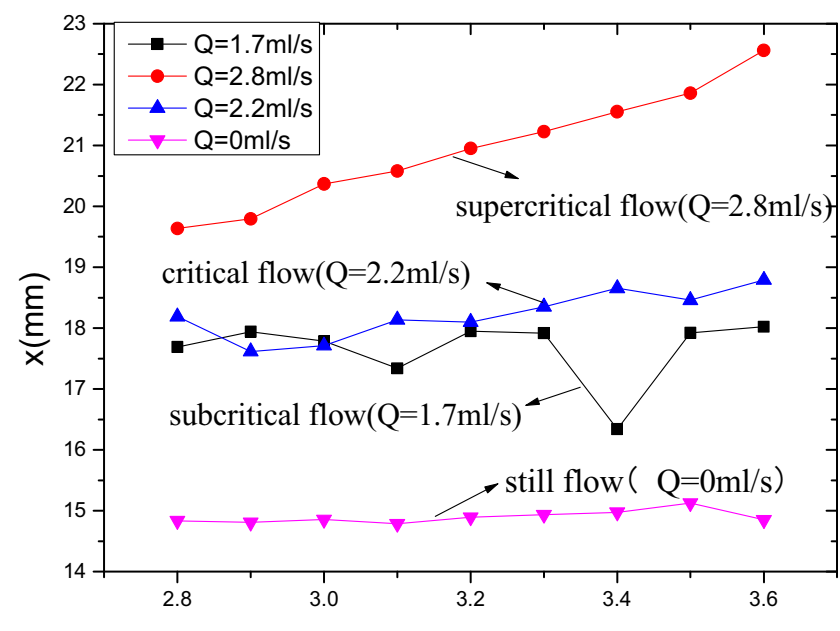

$\mathrm{t}(\mathrm{s})$

Fig. 7 Variation of the location of lowest point (x direction) of free surface during the microgravity time, for symmetrical channel 


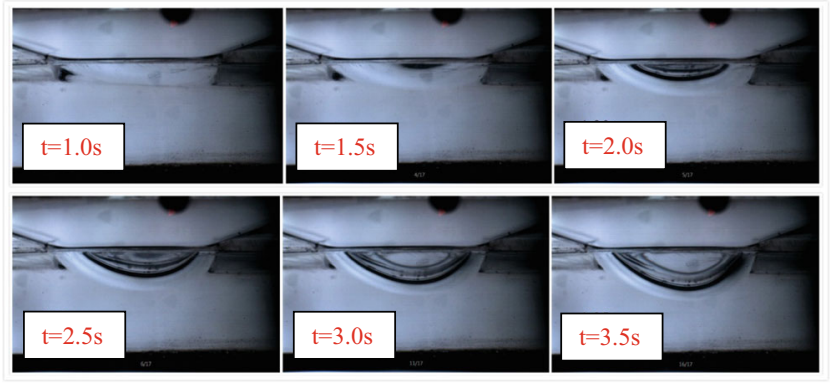

Fig. 8 Typical images of the critical capillary flow in the asymmetrical channel, $\mathrm{Q}=2.7 \pm 0.2 \mathrm{ml} / \mathrm{s}$, flow direction: from left to right

lowest points of the free surface oscillates in $\mathrm{x}$ direction and hardly move forward to the outlet, the flow pattern is in the case of the subcritical capillary flow. When the flow rate is higher than the critical value, the points of the free surface oscillate slightly and displace straight forward to the outlet, the flow pattern becomes the supercritical capillary flow. Between above two patterns and the flow rate near the critical flow, the lowest points of free surface oscillate and move forward very slowly, the flow pattern corresponds to the critical capillary flow. The evolutions of the configuration of free surface when the flow rate reached the critical value are shown in Fig. 8. for the asymmetrical channel and Fig. 9 for the symmetrical channel, respectively.

Whereas in y direction, the location of lowest points of the free surface of capillary flow in the asymmetrical channel declines faster than that in the symmetrical one, as shown in Fig. 10.

If the flow rate is much higher than the critical value, the location of lowest points of the free surface of capillary

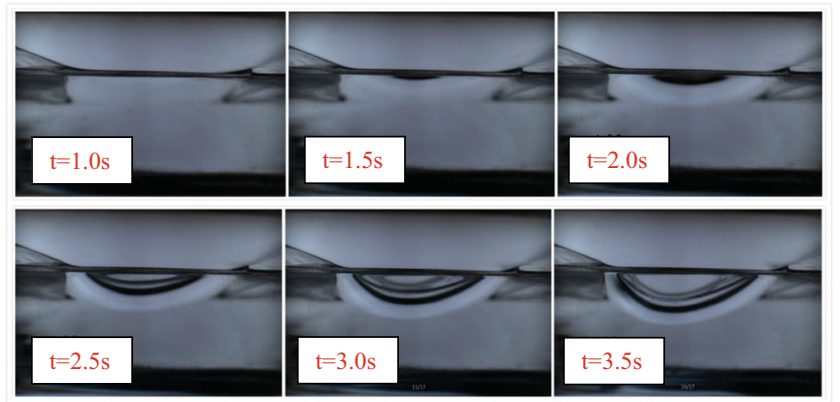

Fig. 9 Typical images of the critical capillary flow in the symmetrical channel, $\mathrm{Q}=2.2 \pm 0.2 \mathrm{ml} / \mathrm{s}$, flow direction: from right to left

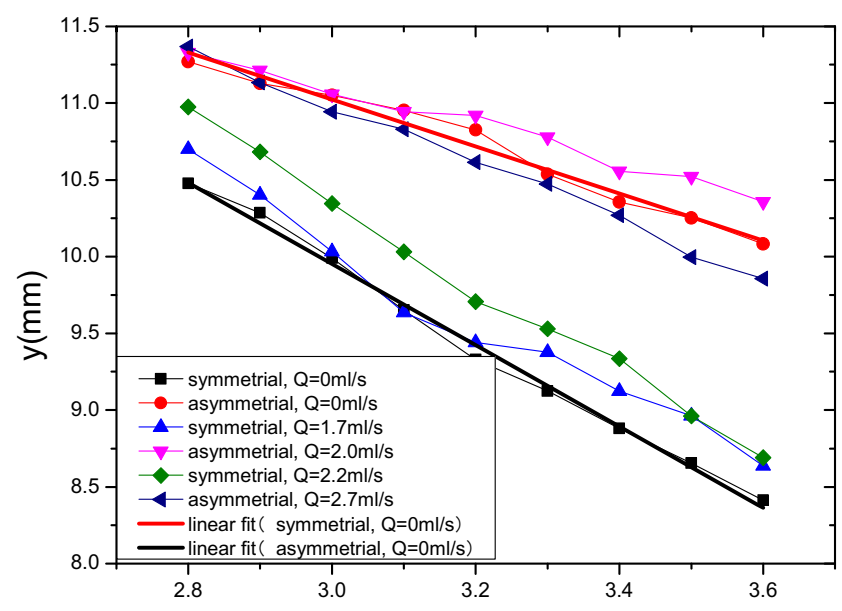

$\mathrm{t}(\mathrm{s})$

Fig. 10 The variation of the location of lowest points of free surface versus time in y direction for two different channels

flow moves forward exponentially in the flow direction, for example, as shown in Fig. 11 for the capillary flow in asymmetrical channel. When the flow rate gets much higher than the critical value $\mathrm{Q}=5.3 \mathrm{ml} / \mathrm{s}$, which is nearly two times of critical flow rate in the microgravity time, we can see the free surface bent increasingly inward, creating fingerlike intrusions that will eventually pinch off ingesting bubbles into the liquid flow, as shown in Fig. 12.

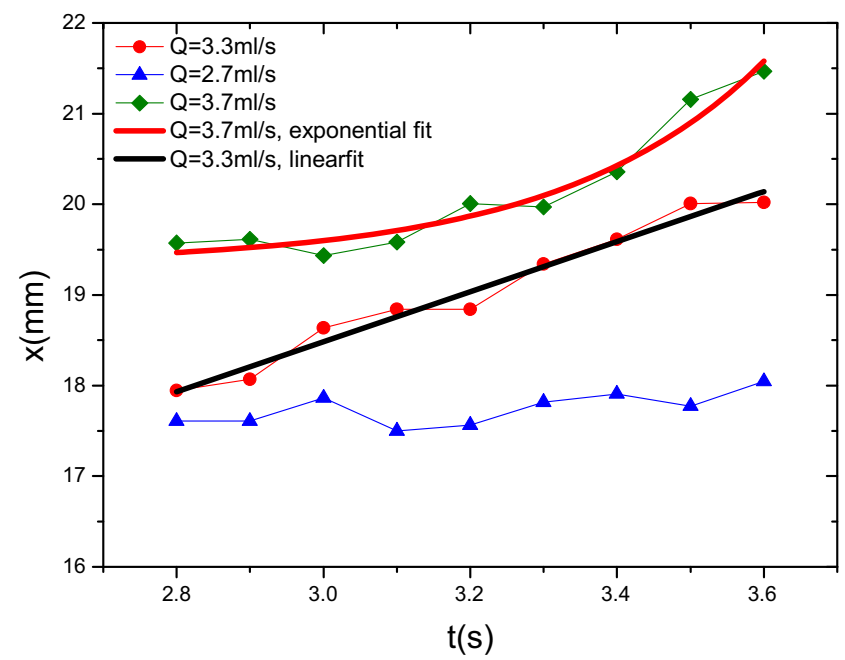

Fig. 11 The variation of the location of lowest points of free surface versus time in $\mathrm{x}$ direction for the asymmetrical channel 


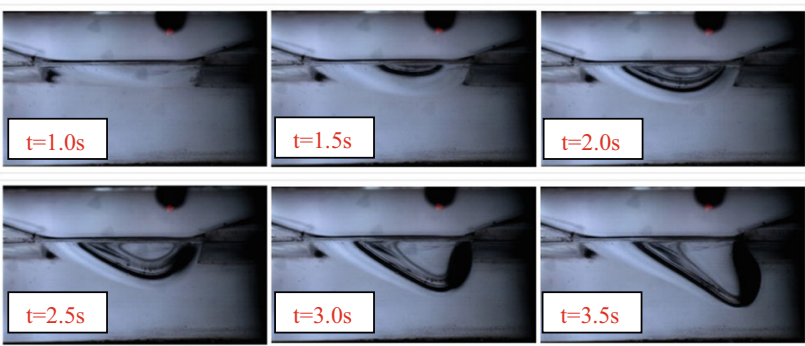

Fig. 12 Typical images showing the high-flow-rate flow in the asymmetrical channel, for $\mathrm{Q}=5.3 \pm 0.2 \mathrm{ml} / \mathrm{s}$, flow direction: from left to right

\section{Conclusions}

The microgravity fluid transport experiment set-up with two capillary channels is developed for the experimental investigation of capillary channel flow by using drop tower. The capillary channel flow has three typical flow patterns: subcritical flow, supercritical flow and critical flow in both the symmetrical channel and asymmetrical channel. For subcritical flow, the lowest points of the free surface oscillates in $\mathrm{x}$ direction and hardly move forward to the outlet, and for supercritical flow, the points of the free surface oscillate slightly and displace straight forward to the outlet, between above two patterns and the flow rate near the critical flow, the lowest points of free surface oscillate and move forward very slowly. The critical flow rates of the free surface of capillary channel flow in microgravity were found experimentaly in two different channels. The critical flow rate of asymmetrical channel is $2.7 \pm 0.2 \mathrm{ml} / \mathrm{s}$, for symmetrical channel is $2.2 \pm 0.2 \mathrm{ml} / \mathrm{s}$. The cross-sectional geometries affect the capillary channel flow behavior. When the flow rate is higher than the critical flow rate, the free surface of capillary flow moves forward exponentially in the flow direction, and the free surface have a tendency to collapse and gas ingestion into the liquid flow.

Acknowledgments This research was financially supported by National Natral Science Foundation of China (Grants No.11532015),
China National High-tech R\&D Program and the Strategic Priority Research Program on Space Science, the Chinese Academy of Sciences

\section{References}

Rollins, J.R., Grove, R.K., Jaekle, D.E.: Twenty-three years of surface tension propellant management system design, development, manufacture, test, and operation. AIAA Paper No, 85-1199 (1985)

Haeberle, S., Zengerle, R.: Microfluidic platforms for lab-on-a-chip applications. Lab Chip 7, 1094-1110 (2007)

Jaekle, D.E.: Propellant management device conceptual design and analysis: Vanes. AIAA Paper No, 91-2172 (1991)

Rosendahl, U., Ohlhoff, A., Dreyer, M.E.: Investigation of forced liquid flows in open capillary channels. Microgravity sci. Technol. 4, 53-60 (2002)

Conrath, M., Canfild, P.J., Bronowicki, P.M., Dreyer, M.E., Weislogel, M.M., Grah, A.: Capillary channel flow experiments aboard the international space station. Phys. Rev. E 88, 1-8 (2013)

Haake, D., Rosendahl, U., Ohlhoff, A.: Flow rate limitation in open capillary channel flows. Interdisciplinary Transport Phenomena in the Space Sciences 1077, 443-458 (2006)

Klatte, J.: Capillary Flow and Collapse in Wedge-Shaped Channels. University of Bremen, Bremen, Germany (2011)

Canfild, P.J., Bronowicki, P.M., Chen, Y., Kiewidt, L., Grah, A., Klatte, J., Jenson, R., Blackmore, W., Weislogel, M.M., Dreyer, M.E.: The capillary channel flow experiments on the international space station: Experiment set-up and fist results. Exp. Fluids 54, 1-14 (2013)

Rosendahl, U., Ohlhoff, A., Dreyer, M.E.: Choked flows in open capillary channels: Theory, experiment and computations. J. Fluid Mech. 518, 187-214 (2004)

Rosendahl, U., Grah, A., Dreyer, M.E.: Convective dominated flows in open capillary channels. Phys. Fluids 22, 1-13 (2010)

Wei, Y.X., Chen, X.Q., Huang, Y.Y.: Flow rate limitation in open wedge channel under microgravity. Sci. China Phys. Mech. Astron. 56(8), 1551-1558 (2013)

Haake, D., Klatte, J., Grah, A., Dreyer, M.E.: Flow rate limitation of steady convective dominated open capillary channel flows through a groove. Microgravity Science And Technology 22, 129-138 (2010)

Grah, A., Dreyer, M.E.: Dynamic stability analysis for capillary channel flow: One-dimensional and three-dimensional computations and the equivalent steady state technique. Phys. Fluids 22, 1-11 (2010)

Bronowicki, P.M., Canfild, P.J., Grah, A., Dreyer, M.E.: Free surfaces in open capillary channels-Parallel plates. Phys. Fluids 27, 1-21 (2015) 